\title{
Low testosterone levels are associated with endothelial dysfunction in oophorectomized early postmenopausal women
}

\author{
Ciciliana Maíla Zilio Rech ${ }^{1}$, Ruth Clapauch ${ }^{1,2}$, Maria das Graças Coelho de Souza ${ }^{1}$ and \\ Eliete Bouskela ${ }^{1}$ \\ ${ }^{1}$ Laboratory for Clinical and Experimental Research on Vascular Biology (BIOVASC), Biomedical Center, \\ State University of Rio de Janeiro, São Francisco Xavier Street, 524. Pavilhão Haroldo Lisboa, BIOVASC, UERJ, \\ Rio de Janeiro, Brazil and ${ }^{2}$ Hospital da Lagoa, Jardim Botânico Street, 501, Endocrinology Sector, Health Ministry, \\ Rio de Janeiro, Brazil
}

\author{
Correspondence \\ should be addressed \\ to C M Z Rech \\ Email \\ cicilianazr@gmail.com
}

\section{Abstract}

Background: The actual consequences of low testosterone levels in women remain uncertain.

Objective: To assess endogenous testosterone influence on body composition, vascular and metabolic function in recent postmenopausal women.

Design: We studied 81 postmenopausal women under transdermal estradiol $\left(E_{2}\right)$ replacement therapy, 36 with bilateral oophorectomy (group O), and 45 controls (group C) through venous occlusion plethysmography, bioimpedance, DEXA, biochemical, hormonal, and inflammatory profile.

Results: Total testosterone level (TT) in group O was $11.0(4.0-17.75)$ vs $23.0(10.0-42.5) \mathrm{ng} / \mathrm{dl}$ in group $\mathrm{C}(P=0.001)$. Forearm blood flow, in $\mathrm{ml} / \mathrm{min} / 100 \mathrm{ml}$ tissue, was lower in group O compared to group $\mathrm{C}$ at baseline (1.57 (1.05-2.47) vs 2.19 (1.592.66) $P=0.036)$, following reactive hyperemia response (endothelium-dependent flow mediated dilatation, 3.44 (2.38-4.35) vs 4.3 (3.09-5.52), $P=0.031$ ) and following nitroglycerin (endothelium-independent dilation, 1.39 (0.99-1.7) vs 1.76 (1.15-2.0), $P=0.025)$, with a positive correlation between TT and all parameters except for the reactive hyperemia response $(r=0.233-$ $0.312, P=0.036-0.004)$. The sVCAM1 levels were negatively correlated with TT $(r=-0.320, P=0.005)$. $E_{2}$ and other hormone levels, biochemical parameters and body composition did not differ between groups. Multiple linear regressions showed that the levels of TT, compared with other confounding variables, may explain the variation observed on endothelial parameters, with low explanatory power.

Conclusion: The absence of ovarian testosterone production in recent postmenopausal oophorectomized women was associated with deleterious effects on endothelial function.

\section{Introduction}

A pronounced decline in estradiol $\left(\mathrm{E}_{2}\right)$ levels follows menopause, a known risk factor for cardiovascular diseases (1). Data from experimental and clinical studies have shown a relationship between estrogen decay and endothelial dysfunction (2), the first and most precocious marker of atherosclerotic process, (3). Testosterone levels show less pronounced descent (2-4 times), compared with estrogens (16 times) (4) because postmenopausal ovaries continue to produce testosterone.
This phenomenon elicits a relative hyperandrogenism in women who experience natural menopause. Oophorectomized women $(\mathrm{O})$, on the other hand, go through a different scenario, in which both $\mathrm{E}_{2}$ and testosterone decline occurs abruptly.

The actual ovarian role after natural menopause and the consequences of low testosterone levels in oophorectomized women, especially regarding vascular health and body composition, have not been thoroughly studied.

Published by Bioscientifica Ltd. 
This subject might influence the decision to perform 'prophylactic' bilateral oophorectomy during hysterectomy, a practice that has increased in the last decades (5), based on concepts that postmenopausal ovaries are not major hormone producing glands and on concerns about risk of ovarian cancer, a type of malignancy usually diagnosed in advanced stages, famed by difficult screening (6) and low survival rates (7).

Besides, the interest on testosterone therapy in women with low libido complaints has grown in the last few years, while cardiovascular consequences remain unclear. Our hypothesis is that low levels of testosterone in oophorectomized women, compared with women in natural menopause, could be associated with poor endothelial function. So, in this investigation we aimed to compare oophorectomized to naturally postmenopausal women, all under estrogen therapy, to determine if testosterone produced by early postmenopausal ovaries influence vascular function assessed by venous occlusion plethysmography, a well-validated method that measures blood flow at baseline and after endothelial nitric oxide production during the reactive hyperemia response. We believe that understanding the mechanisms and possible risks and benefits of endogenous testosterone is essential to decide for developing or disregarding testosterone therapies, and to know to which postmenopausal women they should be considered.

\section{Subjects and methods}

\section{Study population}

Eighty-one postmenopausal patients were studied, 36 with bilateral oophorectomy from etiology other than hormonedependent cancer (group $\mathrm{O}$ ) and 45 with both ovaries (group C), all with no more than 10 years of hypoestrogenism, and adequately compensated from climacteric symptoms by estrogen therapy.

Exclusion criteria were unilateral oophorectomy, contraindications to hormone therapy (previous or current history of breast or endometrial cancer; vaginal bleeding of unknown cause; serious or recent hepatobiliary disease; and thromboembolic, cerebral, or heart ischemic disease), history of polycystic ovary syndrome, or severe acne or hirsutism in reproductive years; users of glucocorticoids, antiandrogenic agents, phytoestrogens, smokers; diabetes using oral hypoglycemic agents or insulin, hypertension not adequately controlled with medication or use of calcium channel blockers or decompensated hyper or hypothyroidism.
Patients were recruited through sample convenience from menopause clinics at Hospital Federal da Lagoa (HFL) and Instituto Estadual de Diabetes e Endocrinologia (IEDE) during their routine appointment with a doctor. The objectives, exams, risks, and benefits of the investigation were explained and those who agreed to participate signed the Term of Consent Agreement for the research project approved by IEDE Ethics Committee (01/2013).

Women taking oral estrogen had their treatment modified to percutaneous $\mathrm{E}_{2}$ gel (Estreva gel) for at least 1 month, in a dose equivalent to prior therapy, while those already in use of $\mathrm{E}_{2}$ gel maintained the same medication and dose. When symptoms of hypoestrogenism were properly compensated (based on clinical evaluation, by patient-oriented subjective score of hot flashes equal or $<3$ in a scale from 0 to 10 ) (8), they initiated the study procedures. The ones not compensated had their $\mathrm{E}_{2}$ gel dose adjusted and were monthly evaluated until they could start. If the patient had uterus and was using a progestin, it was temporarily interrupted, no longer than 3 months, to standardize the hormone therapy (HT) during the study. These women received $200 \mathrm{mg}$ daily of micronized progesterone for 14 days after the conclusion of the study, when all returned to previous HT prescribed at their original clinics.

\section{Data collection}

Clinically compensated patients were instructed to perform a blood test at IEDE laboratory dosing serum levels of $\mathrm{E}_{2}$, estrone, androstenedione, FSH, TT, SHBG and albumin, DHEAS, complete lipid profile, fasting glucose, and insulin.

Fasting glucose, total cholesterol, triglycerides (TG), and HDL-c levels were analyzed by enzyme-colorimetric assay using Hitachi cobas c 701/702 systems, Roche Diagnostics $\mathrm{GmbH})$. Glucose's detection range was 2-750 mg/dl; intra and inter-assay coefficient of variation (CV) were $<0.9$ and $<1.4 \%$ respectively. Total cholesterol's detection range was $3.86-800 \mathrm{mg} / \mathrm{dl}$; intra and interassay CV were $<0.9$ and $<1.7 \%$ respectively. HDL-c's detection range was $3-120 \mathrm{mg} / \mathrm{dl}$; intra and inter-assay were CV $<0.9$ and $<1.6 \%$ respectively. Triglycerides' detection range was $8.85-885 \mathrm{mg} / \mathrm{dl}$; intra and inter-assay $\mathrm{CV}$ were $<1$ and $<2.1 \%$ respectively. Androstenedione was performed by competitive chemiluminescent enzyme immunoassay (IMMULITE 2000 analyzer, Siemens Medical Solutions Diagnostics, Los Angeles, CA, USA). The detection range was $300-1000 \mathrm{pg} / \mathrm{ml}$; intra and interassay CV were $<11.4$ and $<13.3 \%$ respectively. 
DHEAS was performed by electrochemiluminescence (Elecsys 2010 and cobas e 411, 601 and 602 analyzers, Roche Diagnostics $\mathrm{GmbH})$. Detection range was $0.100-1000 \mu \mathrm{g} / \mathrm{dl}$; intra and inter $\mathrm{CV}$ were $<2.9$ and $<4.8 \%$, respectively. Estrone was analyzed by competitive immunoassay (Diagnostics Biochem Canada, Inc., Ontario, Canada) and the limit detection value was $10 \mathrm{pg} / \mathrm{ml}$; intra and inter $\mathrm{CV}$ were $<9.2$ and $<11.8 \%$ respectively. $\mathrm{E}_{2}$, testosterone, $\mathrm{FSH}$, and insulin were performed by electrochemiluminescence immunoassay (MODULAR ANALYTICS E 170, Elecsys 2010 and cobas e 411,601 and 602 analyzers, Roche Diagnostics $\mathrm{GmbH})$. $\mathrm{E}_{2}$ 's detection range was $5-3000 \mathrm{pg} / \mathrm{ml}$; intra and inter $\mathrm{CV}$ were $<8.6$ and $<12 \%$ respectively. Testosterone's detection range was $0.72-432.2 \mathrm{ng} / \mathrm{dl}$; intra and inter $\mathrm{CV}$ were $<4.6$ and $<4.2 \%$ respectively. Validation tests are routinely performed in our laboratory upon kits opening. For testosterone, 20 samples were compared with determination of Pearson's CV of 0.998, and in all tests the total error was lower than total allowable error for the method. FSH's detection range was $0.1-200 \mathrm{mIU} / \mathrm{ml}$; intra and inter $\mathrm{CV}$ were $<5.4$ and $<2.9 \%$ respectively. Insulin's detection range was $0.2-1000 \mu \mathrm{U} / \mathrm{ml}$; intra and inter $\mathrm{CV}$ were $<5$ and $3.8 \%$ respectively. LDL-C, HOMA IR, free and bioavailable testosterone were calculated $(9,10,11)$ according to well-established methods.

During the same week, in previously scheduled day, patients attended the Laboratory for Clinical and Experimental Research in Vascular Biology (BioVasc) after $12 \mathrm{~h}$ fast, waited for at least $20 \mathrm{~min}$ in an acclimatized room and then proceeded to the examination room, where anthropometric evaluation was performed, comprising height, weight, waist (WC), and hip (HC) circumferences. Blood pressure assessment was carried out by two measurements with 10-min interval, with the mean of both being considered. WC was defined as the average of two measurements made after expiration horizontally at the midpoint between the last rib and iliac crest while $\mathrm{HC}$ was measured at the greatest width of the femoral trochanter (12). Waist hip ratio (WHR) was estimated, dividing WC by HC. Weight was measured with patients in light clothes, without shoes, in a high-precision digital scale (Filizola, São Paulo, SP, Brazil) and height was obtained from a wall stadiometer to calculate BMI, dividing body weight in $\mathrm{kg}$ by the square of height in meters.

Blood samples were collected and plasma stored at $-80{ }^{\circ} \mathrm{C}$ for later analyses of inflammatory biomarkers (adiponectin, lipocalin-2/NGAL (neutrophil gelatinaseassociated lipocalin), adipsin and Plasminogen Activator
Inhibitor 1 (PAI1) that were dosed by Milliplex, using MAP-Human Panel Adipokine Magnetic Bead 1 kit, reference HADK1MAG-61K (EMD Milllipore Corporation, Billerica, MA, USA). The sample dilution factor was 1:400; with CV for intra and inter-assay of $<3.75$ and $3.49 \%$ respectively. GDF15 levels (Growth Differentiation Factor 15), MPO (Myeloperoxidase), Myoglobin, P-selectin (Platelet selectin) and Soluble Vascular Cell Adhesion Molecule-1 (sVCAM1) were evaluated by MAP kit Milliplex-Human cardiovascular disease (CVD) Panel 2 Magnetic Bead Kit, reference HCVD2MAG-67K, with dilution factor of 1:100, intra and inter-assay CV\% $<5.33$ and $<7.79 \%$ respectively. All tests were conducted using simultaneous analysis of multiple analytes in magnetic format; concentration of biomarkers was determined by correlation between fluorescence value of samples to ones used for standard curves generated by logistic fit, by data analysis program (Milliplex Analyst Software Vigene Tech EMD Millipore, Billerica, MA, USA).

After collecting blood, patient's regional body composition and differentiated lean, fat and bone tissue components were evaluated at the Nutrition Evaluation Laboratory, UERJ, by (13) dual energy X-ray absorptiometry densitometry (DEXA, GE Healthcare, Lunar iDXA, precision of $0.9 \%$ for fat percentage, $0.8 \%$ for fat mass and $0.5 \%$ for lean mass) (14). The percentage of body fat was evaluated in total, as well as in android (A) and gynoid (G) distribution. Other evaluated parameters were fat mass, total lean mass and $A / G$ rate. Adequate position of the patient at the scanner table, proper hydration status, fasting for solids and light clothing were observed (15).

Back to BioVasc, body composition was also analyzed by tetrapolar bioelectrical impedance to discriminate total content of lean and fat mass, by different impedances that water-rich (muscles, organs) or poor tissues (fat) have to electrical flow. Distal and proximal electrodes were fixed on the dorsal surface of the hands and feet with the individual in supine position (16). A low intensity alternating current (between 500 and $800 \mathrm{~mA}$ ) originates from emitting electrodes and the current difference, caused by resistance and reactance, outline tissue impedance values.

Finally, patients underwent venous occlusion plethysmography of the brachial artery, a non-invasive method to study vascular function through baseline flows, response to reactive hyperemia (endothelium-dependent vasodilatation) and nitroglycerin (endothelium-independent vasodilatation). Briefly, patients were accommodated in the supine position at the examination room, with temperature between 22 and $25^{\circ} \mathrm{C}$. Two pneumatic cuffs, 
connected to Hokanson plethysmograph system (Bellevue, DC, USA), were placed, one around the left arm and another around the left wrist to isolate forearm circulation, as well as an elastic stretch mercury sensor, positioned at the largest circumference of the left forearm. Blood pressure was measured in the contralateral arm and heart rate continuously monitored by electrodes placed on the chest connected to the plethysmograph. The examination began with the reading of baseline flow rate (BF1). For ischemia, the arm cuff was inflated to $200 \mathrm{mmHg}$ for $5 \mathrm{~min}$ and then deflated (17). Within $10 \mathrm{~s}$, the cuff was inflated again, now to $50 \mathrm{mmHg}$. This pressure, higher than venous pressure and lower than diastolic blood pressure, causes selective interruption of venous flow and allows increased forearm blood volume in response to release from ischemia, similar to what occurs with physical exercise, since flow increases after ischemia (shear stress) stimulates endothelial release of nitric oxide. Flow measurements during the reactive hyperemia (RHF) response were recorded $10 \mathrm{~s}$ after ischemia release and during $2 \mathrm{~min}$. The procedure was followed by $15-\mathrm{min}$ interval and new baseline measurement (BF2). Subsequently, the patient received sublingual nitroglycerin (Nitrolingual BurnsAdler Pharmaceuticals, Inc., Charlotte, NC, USA), at a dose of $400 \mu \mathrm{g}$ and after 5 min reactive nitroglycerine flow (RNF) measurement was performed to evaluate vascular response to a nitric oxide donor and, thus, the integrity of the vascular wall, which, together with endothelial function estimated by RHF, provide consistent data of global vascular function.

After plethysmography, patients received a snack and were released with recommendation to return to HT previously prescribed at their original clinic.

\section{Statistical analysis}

The descriptive analysis was expressed as median and interquartile range (25-75\%), according to: i) comparison of clinical and laboratory variables between groups $\mathrm{O}$ and $C$ by Student $t$-test for independent samples or MannWhitney test for numerical data and chi-square $\left(\chi^{2}\right)$ or Fisher exact tests for categorical data and ii) association between numeric variables by Spearman correlation coefficient. Nonparametric methods were applied, due to dispersion of data and rejection of normality hypothesis of Shapiro-Wilks test. Multiple linear regression analysis was performed to assess simultaneous influence of testosterone in presence of confounding variables on endothelial function measurements. Variable selection process was 'stepwise forward' at the level of 5\%. Logarithmic transformation was applied on endothelial function measurements (dependent variables) to homogenize variance. Explanatory variables considered were: testosterone levels, hormone replacement time, hypoestrogenism time, BMI, waist circumference, DEXA, and $\mathrm{E}_{2}$ values. Statistical analyses were done with the Software SAS System, version 6.11 (SAS Institute, Inc., Cary, North Carolina, USA) and 5\% was the level of significance.

\section{Results}

The whole sample comprised 81 postmenopausal women, median age $53.0(50.0-56.5)$ years, who started HT at 51.0 (47.0-53.0) years, had maximum of 10 years of hypoestrogenism and BMI of $28.0(25.9-32.7) \mathrm{kg} / \mathrm{m}^{2}$. When compensated, after at least a month of using percutaneous estrogen, their serum $\mathrm{E}_{2}$ levels were $52.7 \mathrm{pg} / \mathrm{ml}$ (from median applied $\mathrm{E}_{2}$ dose of $1 \mathrm{mg}$ ), $\mathrm{FSH} 47.86 \mathrm{mIU} / \mathrm{ml}$, SHBG $54.0 \mathrm{nmol} / 1$, and DHEAS $65.5 \mu \mathrm{g} / \mathrm{dl}$.

Thirty-six patients had undergone bilateral oophorectomy for benign diseases, at 47.0 (41.3-50.0) years (group O), while 45 kept their two ovaries (group C).

The groups did not differ in age, BMI, WC, WHR, blood pressure, and heart rate (Table 1). However, group $\mathrm{O}$ had less time of hypoestrogenism than group C (12.0 (6.0$48.0)$ vs 36.0 (12.0-60.0) months, $P=0.046)$, reflecting earlier HT use in women that removed both ovaries. Although both groups had similar serum $\mathrm{E}_{2}$ levels, the dose of $\mathrm{E}_{2}$ gel needed to control climacteric symptoms was higher in group $\mathrm{O}$ compared with group $\mathrm{C}$ (1.5 vs $1 \mathrm{mg}$, $P=0.023)$. Group $\mathrm{O}$ had fewer years of education than group C (8.0 (6.0-11.0) vs 11.0 (8.0-11.0), $P=0.028)$.

Testosterone levels differed between groups: TT was 11.0 (4.0-17.75) in group O and 23.0 (10.0-42.5) ng/dl in group $\mathrm{C}(P=0.001)$. The same occurred with calculated free $(0.136(0.046-0.236)$ vs $0.350(0.121-0.603) \mathrm{ng} / \mathrm{dl}$, $P=0.001)$, and bioavailable testosterone levels (3.21 (1.06$5.84)$ vs $8.2(2.97-14.6) \mathrm{ng} / \mathrm{dl}, P=0.001)$. No statistically significant differences were found for other hormonal and metabolic variables (Table 1 ).

All parameters evaluated by venous occlusion plethysmography (BF1, RHF, BF2, and RNF) were significantly lower for group O compared with group C (Fig. 1).

The groups did not differ in body composition, either by densitometry through DEXA or bioimpedance (Table 2) nor in inflammatory biomarkers (Table 3).

Testosterone levels showed positive correlations with vascular flow at all stages of plethysmography except for the reactive hyperemia response $(r=0.233-0.312$, 
Table 1 Clinical, hormonal and biochemical features of the groups. Data are presented as median and interquartile range (25-75\%).

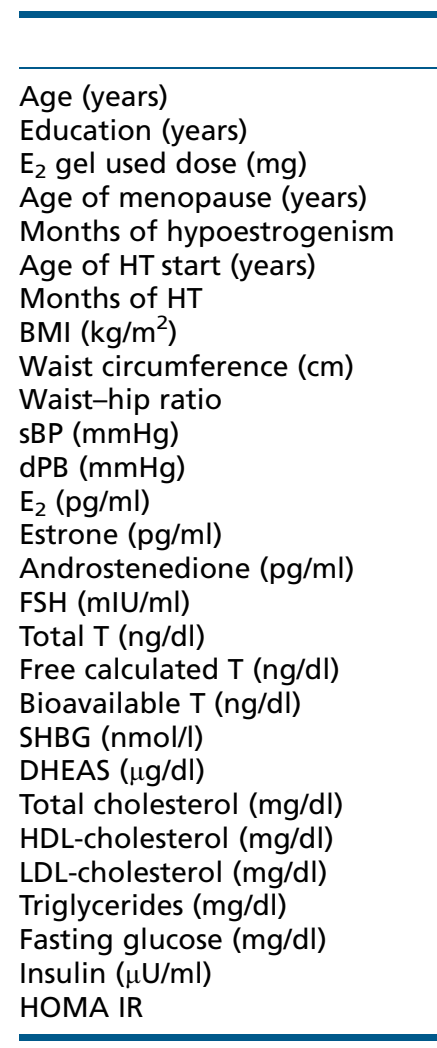

\begin{tabular}{c}
\hline Group 0 \\
\hline $53.0(49.0-57.0)$ \\
$8.0(6.0-11.0)$ \\
$1.5(1.0-1.5)$ \\
$47.0(41.3-50.0)$ \\
$12.0(6.0-48.0)$ \\
$49.0(45.3-52.8)$ \\
$24.0(1.0-105.0)$ \\
$27.6(26.5-32.6)$ \\
$93.5(84.5-98.9)$ \\
$0.881(0.828-0.920)$ \\
$127.5(118.3-134.0)$ \\
$74.5(71.3-78.8)$ \\
$59.1(20.0-103.5)$ \\
$85.0(64.0-145.0)$ \\
$569.0(392.5-1132.5)$ \\
$51.2(26.6-63.8)$ \\
$11.0(4.0-17.75)$ \\
$0.1355(0.046-0.246)$ \\
$3.205(1.06-5.84)$ \\
$54.85(33.1-68.9)$ \\
$56.5(41.3-93.3)$ \\
$212.0(186.5-241.3)$ \\
$60.71(54.7-76.6)$ \\
$115.0(91.3-153.5)$ \\
$114.0(89.0-143.0)$ \\
$96.0(89.3-102.0)$ \\
$7.035(5.04-10.05)$ \\
$1.755(1.16-2.46)$ \\
\end{tabular}

\begin{tabular}{c} 
Group C \\
\hline $53.0(50.0-56.0)$ \\
$11.0(8.0-11.0)$ \\
$1.0(1.0-1.0)$ \\
$48.0(46.0-51.0)$ \\
$36.0(12.0-60.0)$ \\
$51.0(48.5-53.0)$ \\
$1.0(1.0-21.0)$ \\
$28.01(25.3-32.9)$ \\
$93.0(86.5-101.75)$ \\
$0.878(0.826-0.911)$ \\
$127.0(111.5-137.5)$ \\
$76.0(79-82)$ \\
$50.98(20.90-85.16)$ \\
$94.0(53.0-137.4)$ \\
$800.0(400.0-1315.0)$ \\
$40.1(21.59-60.65)$ \\
$23.0(10.0-42.5)$ \\
$0.35(0.1215-0.6025)$ \\
$8.2(2.97-14.6)$ \\
$52.7(35.8-67.45)$ \\
$92.0(47.5-114.0)$ \\
$203.0(179.5-224.0)$ \\
$62.0(52.0-77.5)$ \\
$115.71(100.51-138.4)$ \\
$103.0(76.5-143.5)$ \\
$91.5(83.5-100)$ \\
$7.2(4.22-9.9)$ \\
$1.73(0.99-2.22)$ \\
\end{tabular}

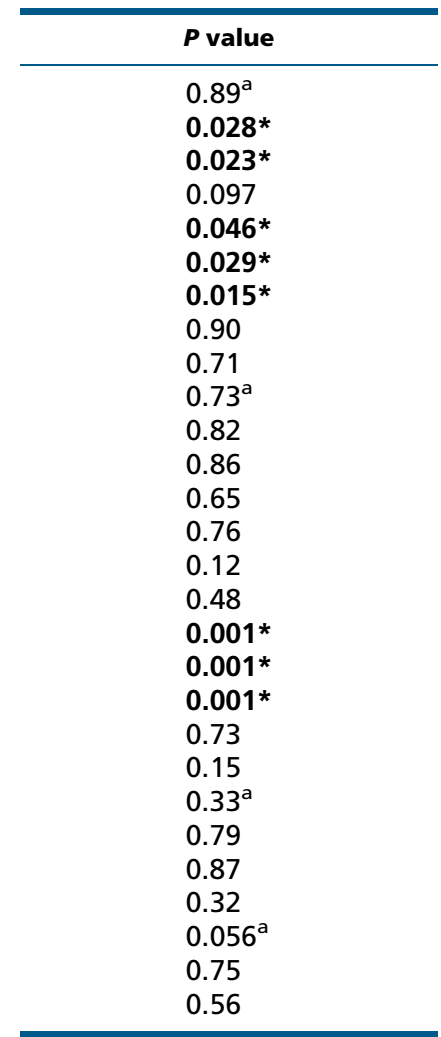

$\mathrm{HT}$, hormone therapy; sBP, systolic blood pressure; dBP, diastolic blood pressure; T, testosterone. Reference values: $\mathrm{E}_{2}<55 \mathrm{pg} / \mathrm{ml}$ (post menopause) and $>45 \mathrm{pg} / \mathrm{ml}$ (under HT); Estrone 10-60 pg/ml; androstenedione 300-3700 pg/ml; FSH > $25.8 \mathrm{mlU} / \mathrm{ml}$ (post menopause) and 3.5-12.5 mlU/ml (under HT); total T 3-63 ng/dl; free calculated T 0.01-1.07 ng/dl; biovailable T 4.4-39 ng/dl; SHBG 16-76 nmol//; DHEAS 35-331 $\mu \mathrm{g} / \mathrm{dl}$ (45-54 years old women), 19-295 $\mu \mathrm{g} / \mathrm{dl}$ (55-64 years old women).

${ }^{a} P<0.05$ by Student's $t$-test or Mann-Whitney test.

$P=0.036-0.004)$ as well as negative correlations with sVCAM1 levels (Table 4).

In a multiple linear regression model, despite an overall low explanatory power, TT levels, compared to other confounding variables, independently explained the variation in RHF $\left(r^{2}=0.054, P=0.004\right)$ and RNF $\left(r^{2}=0.14\right.$, $P=0.025)$. Other variables that showed an independent contribution at the level of $5 \%$ were WC in relation to BF2 $\left(r^{2}=0.075, P=0.016\right)$ and to RNF $\left(r^{2}=0.14, P=0.019\right)$ and time of HT in relation to BF1 $\left(r^{2}=0.073, P=0.018\right)$.

\section{Discussion}

Endothelial dysfunction is an early marker of cardiovascular diseases (18), the leading cause of death in women after 50 years of age (19). The association between hypoestrogenism and endothelial dysfunction is well established (1) as well the beneficial effects of estrogen administration on endothelium (20) in early post menopause.

In this study we have demonstrated, to our knowledge for the first time, that even in recent menopause and under appropriate estrogen replacement, oophorectomized women presented consistent signs of endothelial dysfunction associated with lower levels of endogenous testosterone, compared to women with both ovaries. Endothelial dysfunction was observed at baseline and under endothelium-dependent (reactive hyperemia response) or independent vasodilatation (sublingual nitroglycerin), reflecting respectively decreased bioavailability of nitric oxide (21) associated with increased vascular wall stiffness in group $\mathrm{O}$ (22). Oophorectomized women have higher risk of cardiovascular events according to Women's Health Initiative study (23). Populational studies including menopausal women up to 69 years reported higher cardiovascular risk in those who underwent bilateral oophorectomy before 50 years 


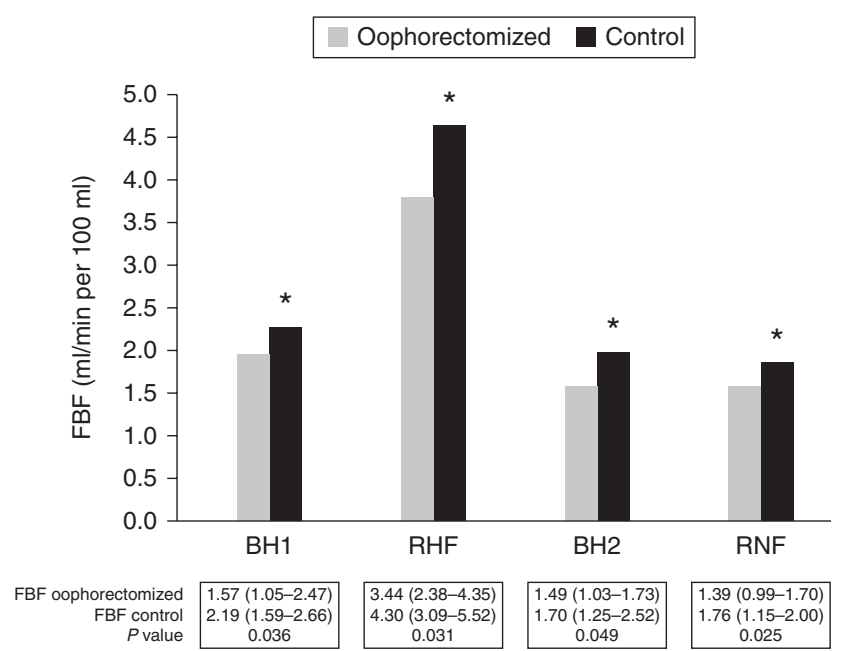

Figure 1

Venous occlusion plethysmography results between groups. $\star P<0.05$ by Mann-Whitney test. FBF, forearm blood flow; $\mathrm{BH}$, baseline flow; RHF, reactive hyperemia.

of age (24), which occurred in 26/35 (almost 75\%) of women in our group O. Montalcini et al. (25) described a positive correlation between serum testosterone levels and flow-mediated brachial artery vasodilatation assessed by Doppler in 60 postmenopausal women. Likewise, Worboys et al. (26), found increased endothelium-dependent and independent vasodilatation after using, associated with estrogen-progestin therapy, $50 \mathrm{mg}$ subcutaneous testosterone implants for 6 weeks.
The mechanisms by which testosterone regulates vascular tone may result from direct androgen receptor mediation or testosterone aromatization into $\mathrm{E}_{2}$, being $\mathrm{E}_{2}$ the activator, via paracrine, of endothelial nitric oxide synthase by non-genomic pathway (27). The aromatase enzyme, responsible for testosterone transformation into $\mathrm{E}_{2}$, was identified in the smooth muscle layer of arteries but not in human endothelial cells (28). However, serum $\mathrm{E}_{2}$ levels were similar between groups, even group $\mathrm{O}$ women requiring larger doses of topical $\mathrm{E}_{2}$ gel preparation to compensate from climacteric symptoms (29), as observed for classical cardiovascular risk factors (age, BMI, WC, glucose, blood pressure, and lipids). Yet, despite the shorter time of hypoestrogenism in group $\mathrm{O}$, given by earlier onset of HT, their vascular response was poor. Endothelial dysfunction in group $\mathrm{O}$ seems to reflect the low endogenous testosterone levels, the only evident potential deleterious difference between the groups, reinforcing the importance of testosterone for proper vascular function in early menopause.

DHEAS and androstenedione, androgens from adrenal and mixed origin (ovarian and adrenal) respectively showed no changes in group O compared with group C, as described by Davison et al. (30) while TT was $10.95 \pm$ 7.49 vs $19.02 \pm 11.53 \mathrm{ng} / \mathrm{dl}$ respectively (30). More recently, Fogle et al. (31) collected samples from ovarian and peripheral veins of women undergoing hysterectomy and demonstrated significant production of ovarian testosterone even 10 years after natural menopause, possibly mediated by high levels of LH in ovarian theca cells.

Table 2 Comparisons of body fat distribution and composition between groups, by anthropometric measurements, bioimpedance and DEXA. Data are presented as median and interquartile range (25-75\%).

\begin{tabular}{l}
\hline \\
\hline BMI $\left(\mathrm{kg} / \mathrm{m}^{2}\right)$ \\
Waist circumference $(\mathrm{cm})$ \\
Waist-hip ratio \\
Bioimpedance \\
LM $(\mathrm{kg})$ \\
FM $(\mathrm{kg})$ \\
LM $(\%)$ \\
FM $(\%)$ \\
DEXA \\
Android fat tissue $(\mathrm{kg})$ \\
Gynoid fat tissue $(\mathrm{kg})$ \\
Total fat tissue $(\mathrm{kg})$ \\
Total fat tissue $(\mathrm{kg})$ \\
Total lean tissue $(\mathrm{kg})$ \\
A/G
\end{tabular}

\begin{tabular}{c} 
Group 0 \\
\hline $27.6(26.5-32.6)$ \\
$93.5(84.5-98.9)$ \\
$0.881(0.828-0.920)$
\end{tabular}

$46.4(41.1-50.1)$

$25.5(20.2-32.2)$

$63.5(59.5-68.0)$

$36.5(32.1-40.5)$

$48.15(43.9-53.4)$

$46.85(42.6-51.0)$

$43.15(39.9-48.4)$

30569 (24 520-37 332)

39942 (34 818-43 246)

$1.05(0.925-1.1)$

\begin{tabular}{ccc}
\hline Group C & & Pvalue $^{*}$ \\
\cline { 1 - 1 } $28.01(25.3-32.9)$ & & 0.90 \\
$93.0(86.5-101.75)$ & & 0.71 \\
$0.878(0.826-0.911)$ & & $0.73^{\mathrm{a}}$ \\
& & \\
$44.6(41.1-52.55)$ & & 0.95 \\
$26.1(20.75-32.0)$ & & 0.90 \\
$62.8(60.05-67.3)$ & & 0.62 \\
$37.2(32.7-39.9)$ & & 0.71 \\
& & 0.22 \\
$49.8(46.7-55.3)$ & & 0.35 \\
$48.55(42.7-51.4)$ & & 0.51 \\
$44.95(41.4-48.1)$ & & 0.81 \\
$30022(24942-38729)$ & 0.78 \\
$37667(35408-43159)$ & $0.90^{\mathrm{a}}$ \\
$1.05(0.97-1.13)$ & &
\end{tabular}

LM, lean mass; FM, fat mass; A/G, android/ gynoid fat tissue.

${ }^{a} P<0.05$ by Student's $t$-test or Mann-Whitney test.

www.eje-online.org 
Table 3 Bio inflammatory markers. Data are presented as median and interquartile range (25-75\%).

\begin{tabular}{|c|c|c|c|}
\hline & Group 0 & Group C & Pvalue* \\
\hline Adiponectin $(\mu \mathrm{g} / \mathrm{ml})$ & $19.34(14.49-37.02)$ & $20.36(11.21-36.55)$ & 0.68 \\
\hline Lipocalin2 (ng/ml) & $79.2(70.3-103.4)$ & 86.2 (73.7-98.8) & 0.79 \\
\hline Adipsin $(\mu \mathrm{g} / \mathrm{ml})$ & $2.91(2.54-3.75)$ & $3.19(2.81-3.73)$ & 0.41 \\
\hline PAl1 (ng/ml) & $48.4(38.76-69.5)$ & $51.2(43.3-65.4)$ & 0.82 \\
\hline GDF15 (ng/ml) & $0.77(0.62-0.93)$ & $0.85(0.62-1.06)$ & 0.28 \\
\hline Myoglobin (ng/ml) & $49.3(38.6-60.9)$ & $46.6(34.4-48.4)$ & 0.066 \\
\hline MPO (ng/ml) & $32.5(22.8-36.9)$ & $28.4(21.9-36.2)$ & 0.41 \\
\hline$P$ selectin (ng/ml) & $198.0(148.0-196.0)$ & $186.0(134.0-264.0)$ & 0.70 \\
\hline sVCAM1 (ng/ml) & $723.0(627.0-828.0)$ & $697.0(590.0-732.0)$ & 0.11 \\
\hline
\end{tabular}

$* P<0.05$ by Mann-Whitney test. PAl1, plasminogen activator inhibitor-1; IGF1/ IGF2, insulin-like growth factor-1/2; GDF15, growth differentiation factor 15; MPO, myeloperoxidase; $P$ selectin, platelet selectin; sVCAM1 soluble vascular cell adhesion molecule-1.

BMI of our sample was $28.0(25.9-32.7) \mathrm{kg} / \mathrm{m}^{2}$, reflecting the current prevalence of overweight worldwide not different between groups $\mathrm{O}$ and $\mathrm{C}$ suggesting that observed endothelial dysfunction could be mediated by androgen levels. McCarty et al. (32), examining oophorectomized women from the National Health and Nutrition Examination Survey (NHANES III) found that fat mass measured by bioimpedance, skinfold thickness, WC and BMI were significantly higher only in women who underwent oophorectomy before 40 years, characterizing premature menopause with stronger associations in those who did not take HT. These results are not comparable to ours: our entire sample was in estrogen replacement and all women were operated after 40 years. Taken together, these studies suggest that low estrogen levels, particularly before age 40, may be higher determinants of body composition than low testosterone levels, being postmenopausal fat more related to low $\mathrm{E}_{2}$ than to testosterone. The lack of difference in adipokine levels (adiponectin, lipocalin2, adipsin) reflects body composition findings, which did not differ between groups. Chalvatzas et al. (33) found similar results for serum levels of adiponectin, comparing women before and after bilateral oophorectomy with or without associated estrogen replacement.

Women with lower testosterone levels (group O) presented higher SVCAM1, endothelial adhesion molecule expressed in atherosclerosis-prone regions and in the periphery of established lesions (34) promoting accumulation and subsequent binding of $\mathrm{T}$ lymphocytes and monocytes to the intima layer (35). In human endothelial cells, testosterone, through its conversion to $\mathrm{E}_{2}$, attenuated atherogenesis by inhibiting VCAM1 expression in a concentration dependent way (36), suggesting that the inflammatory phase of atherosclerotic disease has already started in these early menopause women with short time of hypoestrogenism.

Androgen excess conditions like polycystic ovaries syndrome (PCOS) are associated with an increased risk of insulin resistance, cardiovascular risk factors and subclinical cardiovascular disease (37). However, it is possible that both low (as in oophorectomy) and high (in PCOS, exogenous abuse) testosterone levels increase cardiovascular risk. In women 50-91 years old from the Rancho Bernardo cohort (1984-2004), an age-adjusted analysis showed a 1.62-fold increased risk of incident coronary heart disease (CHD, 95\% confidence interval 1.10-2.39) for the lowest total testosterone quintile compared to higher levels. The lowest and the highest bioavailable testosterone (BT) quintiles were associated with significantly increase age-adjusted risk of incident CHD, with a $79 \%(P=0.046)$ increase risk for women with low BT and a 96\% $(P=0.022)$ increased risk for those with high BT. This $U$-shaped association with incident CHD persisted after additional adjustment for adiposity and lifestyle characteristics (38).

A recent study may evidence the multiple mechanisms that involve testosterone effects on endothelium function. The hyperandrogenism seen in PCOS, rather constituted by testosterone (39), was shown to be linked to increased advanced glycation end-products

Table 4 Correlations of testosterone levels with venous occlusion plethysmography results and sVCAM1 levels.

\begin{tabular}{|c|c|c|c|c|c|c|}
\hline & & BF1 & RHF & BF2 & RNF & sVCAM1 $(\mathrm{ng} / \mathrm{ml})$ \\
\hline \multirow[t]{2}{*}{ Total testosterone (ng/dl) } & $r_{s}$ & 0.235 & 0.184 & 0.233 & 0.312 & -0.320 \\
\hline & $P$ & 0.034 & 0.10 & 0.036 & 0.004 & 0.005 \\
\hline \multirow[t]{2}{*}{ Free calculated testosterone } & $r_{s}$ & 0.206 & 0.144 & 0.227 & 0.311 & -0.344 \\
\hline & $P$ & 0.064 & 0.20 & 0.041 & 0.005 & 0.002 \\
\hline \multirow[t]{2}{*}{ Biovailable testosterone } & $r_{s}$ & 0.205 & 0.148 & 0.219 & 0.299 & -0.357 \\
\hline & $P$ & 0.067 & 0.19 & 0.049 & 0.007 & 0.002 \\
\hline
\end{tabular}

$r_{s}$ Spearman correlation coefficient; $P$ descriptive level. BH, baseline flow rates; RHF, reactive hyperemia flow; RNF, reactive nitroglycerine flow (all expressed in $\mathrm{ml} / \mathrm{min} / 100 \mathrm{ml}$ tissue). 
(AGEs), capable of generating reactive oxygen species and translocation of kappa B nuclear factor, which triggers inflammation, immune response and apoptosis (40). Diamanti-Kandarakis et al. (41), in a cross-sectional study with 106 healthy postmenopausal women, found that those with testosterone levels in the highest quartile (53-160 ng/dl) had significantly higher serum AGEs levels compared to the three quartiles comprising testosterone levels between 2 and $53 \mathrm{ng} / \mathrm{dl}$, even after adjustments for insulin and HOMA-IR. In our study oophorectomized women and those with both ovaries had median testosterone levels comparable to Diamanti-Kandarakis's first (2-23 ng/dl) and second (23-35 ng/dl) quartiles respectively where AGEs were reported to be lower. We excluded women with a history of polycystic ovary syndrome, severe acne or hirsutism in reproductive years; besides, all were on estrogen therapy, which reduces gonadotropins and, thus, ovarian stimulation, which may explain why testosterone levels in our study were all below $42.5 \mathrm{ng} / \mathrm{dl}$; equally, insulin, glucose and HOMA-IR were not different between the groups we analyzed. Therefore, lower postmenopausal testosterone levels may directly depress endothelial nitric oxide (NO) production mediated through the androgen/estrogen receptor, while higher levels increase AGEs and impair NO bioavailability at a post-receptor level. Altogether, these studies suggest that, concerning endothelial function, an optimal range of circulating testosterone may exist, not as low as post oophorectomy neither as high as in PCOs.

Regarding androgen replacement, in a recent randomized study, Huang et al. (42) provided weekly placebo vs 3 , $6.25,12.5$ or $25 \mathrm{mg}$ testosterone enanthate i.m. injections to hysterectomized women with serum total testosterone concentrations less than $31 \mathrm{ng} / \mathrm{dl}$ during 24 weeks, achieving mean on-treatment nadir total concentrations of $14,79,105,130$, and $232 \mathrm{ng} / \mathrm{dl}$ respectively. No significant changes in cardiovascular risk markers such as fasting glucose, fasting insulin, HOMA IR, high sensitivity C-reactive protein, adiponectin, blood pressure, and heart rate were observed in any subgroup. However, the longterm consequences of androgen replacement are not known. Potential masculinizing effects like acne, hirsutism or alopecia are dose related, especially due to supraphysiological hormone levels. Besides, endometrial changes can vary from atrophy to hyperplasia, in a result from peripheral conversion to estrogens.

One of the strengths of our study was to focus on androgen influences in early postmenopausal women, an important phase recognized by the literature. Endothelial dysfunction and inflammatory biomarkers represent gold standard signals of the atherosclerotic process initiation and the great homogeneity between groups $\mathrm{O}$ and $\mathrm{C}$, especially in relation to cardiovascular risk factors, constitutes a major advantage. Furthermore, all women were studied under adequate estrogen replacement, so we could analyze, in fact, the isolated effects of low endogenous testosterone levels in oophorectomized women.

As limitations, data at the time of hypoestrogenism and age of oophorectomy are subject to recall bias. An explorative study carries an innate risk of false positive findings and although we performed a multiple linear regression, other adjustments for multiple tests could not be applied because they are designed for comparisons of more than two groups. Direct measurement of immunoassays for testosterone are susceptible to cross-reaction due to structural similarity between them and methods such as chromatography/mass spectrometry are preferred in groups with low hormone levels, like children an postmenopausal women; however, electrochemiluminescence immunoassay was the only available method for measuring testosterone in our city until very recently (August 2015) and it is still the standard process. Besides, there is a biological plausibility for the lower testosterone levels found in oophorectomized women supported by the population study of Davison et al. (30) who provided normative data for physiological androgen levels in adult females according to age and found significantly lower testosterone levels in oophorectomized compared to age matched women in spontaneous early menopause. Nonetheless, the findings of this study cannot be extrapolated for other androgen imbalance conditions like primary adrenal insufficiency or male hypogonadism.

In conclusion, postmenopausal oophorectomized women, compared with controls, both in early post menopause and under proper estrogen replacement, showed low endogenous testosterone levels associated with poor endothelial function. More studies are needed regarding delaying progression of cardiovascular disease through ovary preservation during gynecological surgery or providing low doses of testosterone to early postmenopausal oophorectomized women.

\section{Declaration of interest}

The authors declare that there is no conflict of interest that could be perceived as prejudicing the impartiality of the research reported.

\section{Funding}

This study was supported by grants from the National Research Council of Brazil (CNPq) and the Agency for Financing Research of the State of Rio de 
Janeiro (FAPERJ). The funders had no role in study design, data collection and analysis, decision to publish, or preparation of the manuscript.

\section{Author contribution statement}

C M Z Rech collected the data and wrote the manuscript. R Clapauch collected the data and wrote/reviewed the manuscript. E Bouskela reviewed/edited the manuscript. M G C de Souza did the inflammatory biomarkers analysis. All authors read and approved the final manuscript.

\section{Acknowledgements}

A special thanks to Rita Vasconcellos Weiss (IEDE) and Georges Sterblitch (Miguel Couto Hospital) for data collection collaboration, to Rosangela Aparecida Martins Noe, from the Federal University of Rio de Janeiro, for statistical analysis, and to Daniel Alexandre Bottino, and Fernando Lencastre Sicuro from BioVasc laboratory, UERJ, for informatics and statistics support respectively.

\section{References}

1 Maturana MA, Irigoyen MC \& Spritzer PM. Menopause, estrogens, and endothelial dysfunction: current concepts. Clinics 200762 77-86. (doi:10.1590/S1807-59322007000100012)

2 Kalantaridou SN, Naka KK, Papanikolaou E, Kazakos N, Kravariti M, Calis KA, Paraskevaidis EA, Sideris DA, Tsatsoulis A, Chrousos GP et al. Impaired endothelial function in young women with premature ovarian failure: normalization with hormone therapy. Journal of Clinical Endocrinology and Metabolism 200489 3907-3913. (doi:10.1210/jc. 2004-0015)

3 Caramori PR \& Zago A. Disfunção endotelial e doença arterial coronariana. Arquivos Brasileiros de Cardiologia 200075 163-172. (doi:10.1590/S0066-782X2000000800009)

4 Davison SL, Bell R, Donath S, Montalto JG \& Davis SR. Androgen levels in adult females: changes with age, menopause, and oophorectomy. Journal of Clinical Endocrinology and Metabolism 200590 3847-3853. (doi:10.1210/jc.2005-0212)

5 Keshavarz H, Hillis SD, Kieke BA \& Marchbanks PA. Hysterectomy surveillance - United States, 1994-1999. Morbidity and Mortality Weekly Report. Surveillance Summaries 200251 1-8.

6 Mai PL, Wentzensen N \& Greene MH. Challenges related to developing serum-based biomarkers for early ovarian cancer detection. Cancer Prevention Research 20114 303-306. (doi:10.1158/1940-6207.CAPR11-0053)

7 Jemal A, Bray F, Center MM, Ferlay J, Ward E \& Forman D. Global cancer statistics. CA A Cancer Journal for Clinicians 201161 69-90. (doi:10.3322/caac.20107)

8 Wygoda M, Filippo RB Jr, Gomes MA \& Clapauch R. Monitorizando a terapia de reposição estrogênica (TRE) na menopausa. Arquivos Brasileiros de Endocrinologia e Metabologia 199943 336-343. (doi:10.1590/S0004-27301999000500005)

9 Friedewald WT, Levy RI \& Fredrickson DS. Estimation of the concentration of low-density lipoprotein cholesterol in plasma, without use of the preparative ultracentrifuge. Clinical Chemistry 1972 18 499-502.

10 Matthews DR, Hosker JP, Rudenski AS, Naylor BA, Treacher DF \& Turner RC. Homeostasis model assessment: insulin resistance and $\beta$-cell function from fasting plasma glucose and insulin concentrations in man. Diabetologia 198528 412-419. (doi:10.1007/BF00280883)

11 Vermeulen A, Verdonck L \& Kaufman JM. A critical evaluation of simple methods for the estimation of free testosterone in serumA critical evaluation of simple methods for the estimation of free testosterone in serum. Journal of Clinical Endocrinology and Metabolism 199984 3666-3672. (doi:10.1210/jcem.84.10.6079)

12 World Health Organ Tech Rep Ser. Obesity: preventing and managing the global epidemic. Report of a WHO consultation. World Health Organization Technical Report Series 2000894 1-253.

13 Bachrach LK. Dual energy X-ray absorptiometry (DEXA) measurements of bone density and body composition: promise and pitfalls. Journal of Pediatric Endocrinology \& Metabolism 200013 983-988.

14 Hangartner TN, Warner S, Braillon P, Jankowski L \& Shepherd J. The official positions of the International Society for Clinical Densitometry: acquisition of dual-energy $\mathrm{X}$ ray absorptiometry body composition and considerations regarding analysis and repeatability of measures. Journal of Clinical Densitometry 201316 520-536. (doi:10.1016/j.jocd. 2013.08.007)

15 Petak S, Barbu CG, Yu EW, Fielding R, Mulligan K, Sabowitz B, Wu CH \& Shepherd JA. The official positions of the international society for clinical densitometry: body composition analysis reporting. Journal of Clinical Densitometry 201316 508-519. (doi:10.1016/j.jocd. 2013.08.018)

16 Kyle UG, Bosaeus I, De Lorenzo AD, Deurenberg P, Elia M, Gómez JM, Heitmann BL, Kent-Smith L, Melchior JC, Pirlich M et al. Composition of the ESPEN Working Group. Bioelectrical impedance analysis - part I: review of principles and methodsComposition of the ESPEN Working Group. Clinical Nutrition 200423 1226-1243. (doi:10.1016/j.clnu.2004. 06.004)

17 Alomari MA, Solomito A, Reyes R, Khalil SM, Wood RH \& Welsch MA. Plethysmography: technical considerations, standardization and physiological findings. American Journal of Physiology. Heart and Circulatory Physiology 2004286 99-107. (doi:10.1152/ajpheart.00529. 2003)

18 Vita JA \& Keaney JF. Endothelial function: a barometer for cardiovascular risk? Circulation 2002106 640-642. (doi:10.1161/01.CIR. 0000028581.07992.56)

19 The World Health Report 2004: Changing History. Geneva, World Health Organization 2004.

20 Clapauch R, Mecenas AS, Maranhão PA \& Bouskela E. Early postmenopausal women with cardiovascular risk factors improve microvascular dysfunction after acute estradiol administration. Menopause 201219 672-679. (doi:10.1097/gme.0b013e31823a8f43)

21 Joannides R, Haefeli WE, Linder L, Richard V, Bakkali EH, Thuillez C \& Lüscher TF. Nitric oxide is responsible for flow-dependent dilatation of human peripheral conduit arteries in vivo. Circulation 199591 1314-1319. (doi:10.1161/01.CIR.91.5.1314)

22 Oliver JJ \& Webb DJ. Noninvasive assessment of arterial stiffness and risk of atherosclerotic events. Arteriosclerosis, Thrombosis, and Vascular Biology 200323 554-566. (doi:10.1161/01.ATV.0000060460.52916.D6)

23 Allison MA, Manson JE, Langer RD, Carr JJ, Rossouw JE, Pettinger MB, Phillips L, Cochrane BB, Eaton CB, Greenland P et al. Women's Health Initiative and Women's Health Initiative Coronary Artery Calcium Study Investigators, oophorectomy, hormone therapy, and subclinical coronary artery disease in women with hysterectomy: the Women's Health Initiative coronary artery calcium study. Menopause $2008 \mathbf{1 5}$ 639-647. (doi:10.1097/gme.0b013e31816d5b1c)

24 Dørum A, Tonstad S, Liavaag AH, Michelsen TM, Hildrum B \& Dahl AA. Bilateral oophorectomy before 50 years of age is significantly associated with the metabolic syndrome and Framingham risk score: a controlled, population-based study (HUNT-2). Gynecologic Oncology 2008109 377-383.

25 Montalcini T, Gorgone G, Gazzaruso C, Sesti G, Perticone F \& Pujia A. Endogenous testosterone and endothelial function in postmenopausal women. Coronary Artery Disease 2007 18 9-13. (doi:10.1097/01.mca. 0000236290.79306.d1)

26 Worboys S, Kotsopoulos D, Teede H, McGrath B \& David SR. Evidence that parenteral testosterone therapy may improve endothelium-dependent and independent vasodilatation in postmenopausal women already receiving estrogen. Journal of Clinical 
Endocrinology and Metabolism 200188 158-161. (doi:10.1210/jcem.86. 1.7103)

27 Wu FCW \& von Eckardstein A. Androgens and coronary artery disease. Endocrine Reviews 200324 183-217. (doi:10.1210/er.2001-0025)

28 Harada N, Sasano H, Murakami H, Ohkuma T, Nagura H \& Takagi Y. Localized expression of aromatase in human vascular tissues. Circulation Research 199984 1285-1291. (doi:10.1161/01.RES.84. 11.1285)

29 Studd J. Variations on hormone replacement therapy: an answer to the 'one dose fits all' Women's Health Initiative study. Gynecological Endocrinology 200723 665-671. (doi:10.1080/09513590701671878)

30 Davison SL, Bell R, Donath S, Montalto JG \& Davis SR. Androgen levels in adult females: changes with age, menopause, and oophorectomy. Journal of Clinical Endocrinology and Metabolism 200590 3847-3853. (doi:10.1210/jc.2005-0212)

31 Fogle RH, Stanczyk FZ, Zhang X \& Paulson RJ. Postmenopausal ovarian androgen production. Journal of Clinical Endocrinology and Metabolism 200792 3040-3043. (doi:10.1210/jc.2007-0581)

32 McCarthy AM, Menke A \& Visvanathan K. Association of bilateral oophorectomy and body fatness in a representative sample of US women. Gynecologic Oncology 2013129 559-564. (doi:10.1016/j.ygyno. 2013.02.016)

33 Chalvatzas N, Dafopoulos K, Kosmas G, Kallitsaris A, Pournaras S \& Messinis IE. Effect of ovarian hormones on serum adiponectin and resistin concentrations. Fertility and Sterility 200991 1189-1194. (doi:10.1016/j.fertnstert.2008.01.027)

34 Cybulsky MI, Iiyama K, Li H, Zhu S, Chen M, Iiyama M, Davis V, Gutierrez-Ramos JC, Connelly PW \& Milstone DS. A major role for VCAM-1, but not ICAM-1, in early atherosclerosis. Journal of Clinical Investigation 2001107 1255-1262. (doi:10.1172/JCI11871)

35 Libby P, Ridker PM \& Maseri A. Inflammation and atherosclerosis. Circulation 2002105 1135-1143. (doi:10.1161/hc0902.104353)
36 Mukherjee TK, Dinh H, Chaudhuri G \& Nathan L. Testosterone attenuates expression of vascular cell adhesion molecule-1 by conversion to estradiol by aromatase in endothelial cells: Implications in atherosclerosis. PNAS 200299 4055-4060. (doi:10.1073/pnas. 052703199)

37 Yilmaz SA, Kebapcilar A, Koplay M, Kerimoglu OS, Pekin AT, Gencoglu B, Dogan NU \& Celik C. Association of clinical androgen excess with radial artery intima media thickness in women with polycystic ovary syndrome. Gynecological Endocrinology 20156 477-482. (doi:10.3109/09513590.2015.1014783)

38 Laughlin GA, Goodell V \& Barrett-Connor E. Extremes of endogenous testosterone are associated with increased risk of incident coronary events in older women. Journal of Clinical Endocrinology and Metabolism 201095 740-747. (doi:10.1210/jc.2009-1693)

39 Livadas S, Pappas C, Karachalios A, Marinakis E, Tolia N, Drakou M, Kaldrymides P, Panidis D \& Diamanti-Kandarakis E. Prevalence and impact of hyperandrogenemia in 1,218 women with polycystic ovary syndrome. Endocrine 201447 631-638. (doi:10.1007/s12020-0140200-7)

40 Pertynska-Marczewska M, Diamanti-Kandarikis E, Zhang J \& Merhi Z. Advanced glycation end products: a link between metabolic and endothelial dysfunction in polycystic ovary syndrome? Metabolism 201564 1564-1573. (doi:10.1016/j.metabol.2015.08.010)

41 Diamanti-Kandarakis E, Lambrinoudaki I, Economou F, Christou M, Piperi C, Papavassiliou AG \& Creatsas G. Androgens associated with advanced glycation end-products in postmenopausal women. Menopause 201017 1182-1187. (doi:10.1097/gme.0b013e3181e170af)

42 Huang G, Tang E, Aakil A, Anderson S, Jara H, Davda M, Stroh H, Travison TG, Bhasin S \& Basaria S. Testosterone dose-response relationships with cardiovascular risk markers in androgen-deficient women: a randomized, placebo-controlled trial. Journal of Clinical Endocrinology and Metabolism 201499 1287-1293. (doi:10.1210/ jc.2013-4160)

Received 30 August 2015

Revised version received 3 December 2015

Accepted 15 December 2015 